\title{
Spectral and photopic studies for high intensity discharge (HID) lamps
}

\author{
A.-E.A. Abd-Elmageed and E.M. El-Moghazy
}

National Institute for Standards (NIS), Photometry and Radiometry Division Tersa St., El- Haram - P.O. Box: 136 Code No.12211, Giza, Egypt

Received: 18 November 2014 / Accepted: 15 January 2015

\begin{abstract}
Outdoor lighting sources such as High Intensity Discharge lamps (HID) are common in these days. This research is focusing on the relation between the spectral power distribution and luminous efficiency function $V(\lambda)$, which describes the average spectral sensitivity of human visual perception of brightness. The most useable HID lamps inside Egyptian markets are high pressure mercury (HPM), high pressure sodium (HPS) and metal halide (MH). A set up based on single monochromator and integrating sphere for relative spectral power distribution and absolute luminous flux measurements are used, respectively. The accompanied standard uncertainties with the measurements are evaluated.
\end{abstract}

Keywords: Outdoor sources, luminous efficiency function, spectral power distribution, Uncertainty

\section{Introduction}

Because the eye varies in its response to different wavelengths of light under differing conditions, true assessment of the lumen output of a lamp should be based on the eye's response under the conditions under which the lamp is used [1]. The high intensity discharge (HID) lamps such as high pressure mercury (HPM), high pressure sodium (HPS) and metal halide (MH) lamps mainly designed for outdoor lighting where we need high efficacy and high luminous flux. Outdoor lighting has several fundamental purposes. It plays a role in crime prevention, improvement of the night time environment, and in providing increased safety. Fundamental issues of visibility relate particularly to security and safety. Enhanced security is provided by improved visibility, and the night driving task is safer if the motorist can see well [2].

High pressure mercury lamps, see Figure 1 [3], have a short quartz glass discharge tube that contains a mixture of inert gas and mercury. As a light source, they are relatively compact, which allows their light to be controlled via optical equipment $[2,4]$. On the other hand, High pressure sodium lamps are the most efficient light source commercially available today; see Figure 2 [3]. They were developed and introduced as energy efficient sources in road lighting, industrial lighting and security application. Nowadays HPS lamps are also appropriate for many interior applications, particularly where colour rendering is not essential concern due to their high efficiency and long life [4]. In the lamp design, the exterior bulb protects the arc tube from changes and drafts in temperature; this

^ Correspondence: emoghazy8@yahoo.com

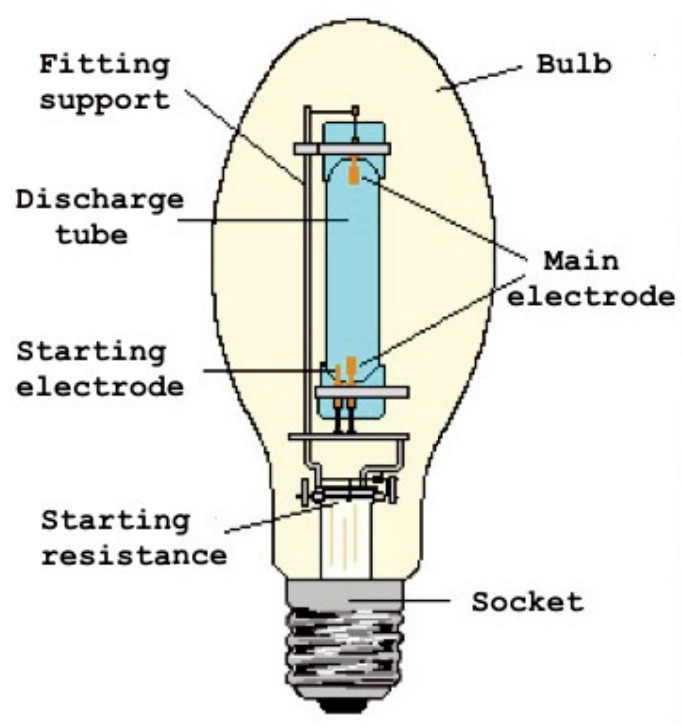

Fig. 1. High pressure mercury lamp [3].

makes HPS lamps especially easy to use in many fixture types $[2,4]$.

Metal Halide lamps, see Figure 3 [5], are a further development of mercury lamps. Apart from mercury, they also contain a mixture of metal halides to improve luminous efficacy and to enhance colour rendering. They have excellent luminous efficacy and good colour rendering qualities; their nominal lamp life is high. They are extremely compact light sources, whose light can be easily controlled $[2,4]$. 


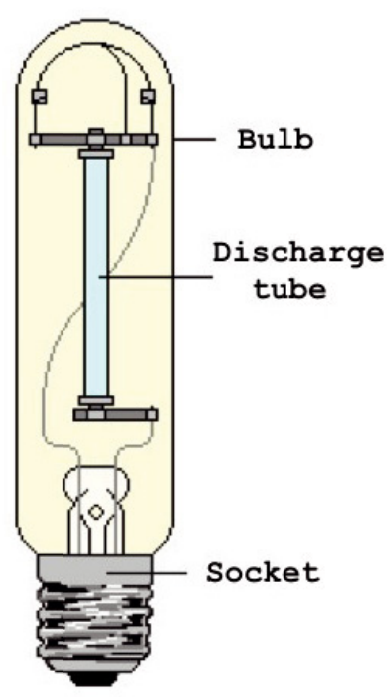

Fig. 2. High pressure sodium lamp [3].

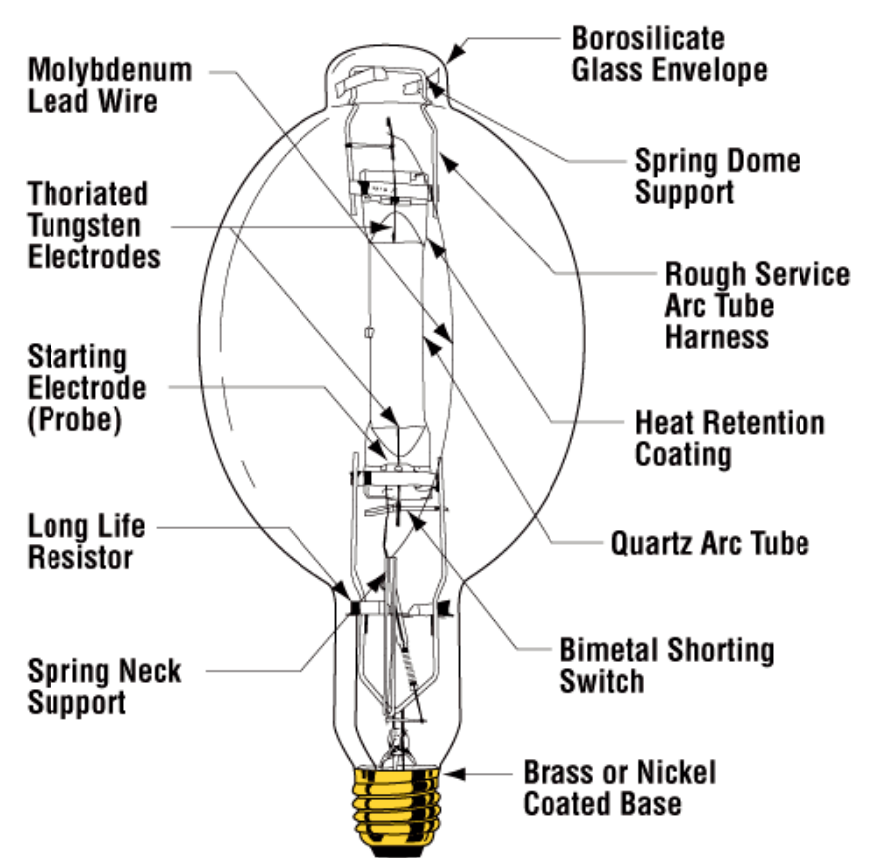

Fig. 3. Metal halide lamp [5].

This research presents the study of three types of HID lamps from OSRAM in terms of their absolute luminous flux and relative spectral power distribution with respect to $V(\lambda)$. Lamps HPM $125 \mathrm{~W}$, HPS $150 \mathrm{~W}$ and MH $150 \mathrm{~W}$ are used as they are safe use in the outdoor lighting. Absolute luminous flux measurements and relative spectral power distribution was studied. Spectral power distribution (SPD) measurement describes the power per unit area per unit wavelength of an illumination (radiant exitance).

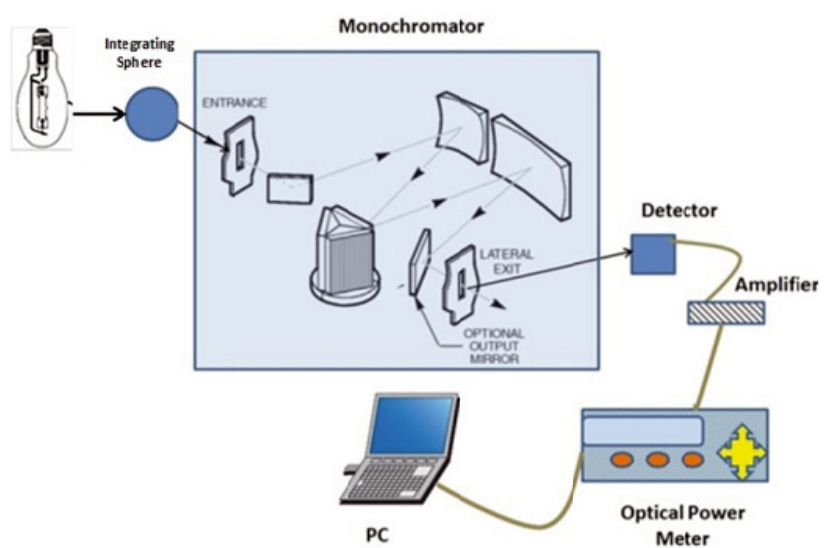

Fig. 4. Schematic diagram of NIS facility for measuring spectral power distribution.

\section{Material and methods}

\subsection{Monochromator-based method}

The setup and monochromator function illustrated in Figure 4 is to measure the radiation from the three spectral lamps produces narrow and intense lines. This radiation is introduced into a small integrating sphere to build a uniform, monochromatic, and nearly Lambertian source of high spectral radiant flux. This integrating sphere of the entrance optics is needed in order to uniformly irradiate the entrance slit of the monochromatic as a function of the irradiance of the source independent from source geometry [6]. In the used spectral range, two 1200 lines $/ \mathrm{mm}$ gratings (blaze wavelengths $250 \mathrm{~nm}$ and $750 \mathrm{~nm}$ ) were used to collect the data of spectral power distribution from the lamps. At the exit slit of the monochromator, a silicon $(\mathrm{Si})$ detector is used to detect the output signal. The lamps was aged according to IES publication [7] then each lamp aligned in front of the integrating sphere entrance slit of a system based on MS-257 single monochromator from Newport Corporation. The system was adjusted to acquire readings at the range from $200 \mathrm{~nm}$ to $800 \mathrm{~nm}$ with step $2 \mathrm{~nm}$, also the monochromator band pass was adjusted nominally at $4 \mathrm{~nm}$ resulting wavelength uncertainty $\pm 0.3 \%$ to $2 \%$ at $k=2[8]$.

\subsection{Integrating sphere based method}

The absolute luminous flux of each lamp is measured using an integrating sphere its diameter $2.5 \mathrm{~m}$. Figure 5 shows the geometry of the NIS $2.5 \mathrm{~m}$ integrating sphere. The integrating sphere is consists of a cosine corrected photometer equipped with $V(\lambda)$ filter from LMT company. The detector has a linearity response with range $16-$ $28 \mathrm{nA} / \mathrm{lx}$, based on these characteristics the total luminous flux from $0.01 \mathrm{~lm}$ to $106 \mathrm{~lm}$ can be measured in direct substitution with total luminous flux of standard lamps of any wattage [9]. The sphere wall is coated with barium sulfate paint with diffuse reflectance approximately $97 \%$ in the visible region. The integrating sphere is also equipped 


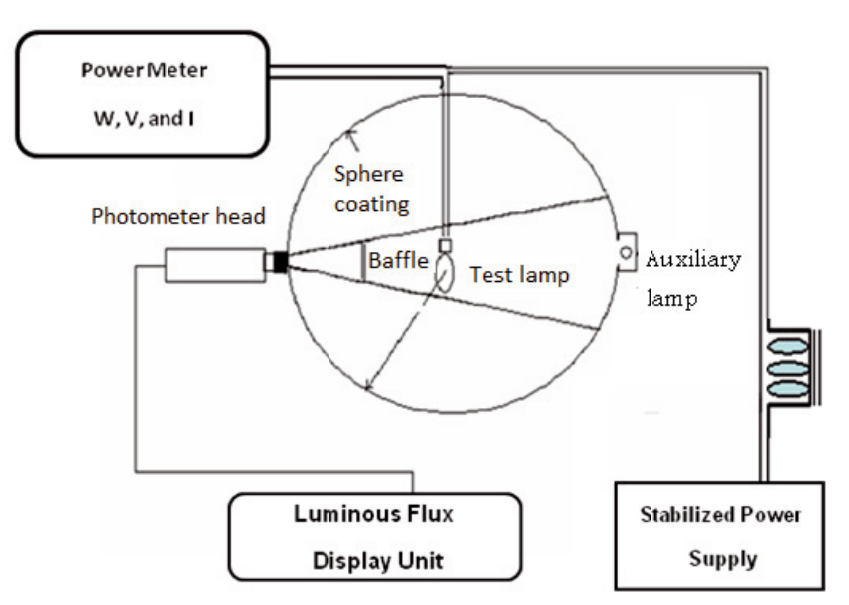

Fig. 5. NIS $2.5 \mathrm{~m}$ integrating sphere set up for absolute total luminous flux.

with auxiliary lamp $(100 \mathrm{~W})$ tungsten on the sphere wall to measure the self-absorption effects of a lamp in the sphere. The room temperature of the photometry laboratory is controlled to be about $24^{\circ} \mathrm{C}$. A temperature sensor is mounted to measure the air temperature of the space inside the sphere. During lamp operation the ambient temperature in the sphere is approximately $25^{\circ} \mathrm{C}[10]$.

\section{Results and discussion}

To determine lamp lumens the power of the light at each wavelength $P(\lambda)$ in the visible spectrum is multiplied by the photopic luminosity function $(V(\lambda))$ value or eye sensitivity at the equivalent wavelengths, according to the International Commission on Illumination (CIE) [11]. The constant $K_{m}$ is a scaling factor called the maximum spectral luminous efficiency for photopic vision and equal to $683 \mathrm{~lm} / \mathrm{W}$ [11]. Then all of these multiplied values are summed to find the lumen output. This may be stated as:

$$
\text { Lamp lumens }=K \int_{380}^{780} P(\lambda) V(\lambda) d \lambda .
$$

The acquired relative spectral power distribution, using the monochromator based method, was normalized for each lamp so we can compare between lamps spectrum (see Fig. 6). Results show HPM, MH and HPS lamps with the comparison of $V(\lambda)$ and how much the relative spectral power of each lamps.

On the other hand, the absolute luminous flux of each lamp in the photopic $V(\lambda)$ range, using integrating based method, is measured using LMT $V(\lambda)$ standard detector. Figure 7 shows results of the acquired absolute luminous flux in lumen from each lamp.

The associated uncertainty must be quoted whenever the results of a measurement are reported. This tells the user of the precision with which the measurement was made. Uncertainty analysis is thus a fundamental part of metrology.

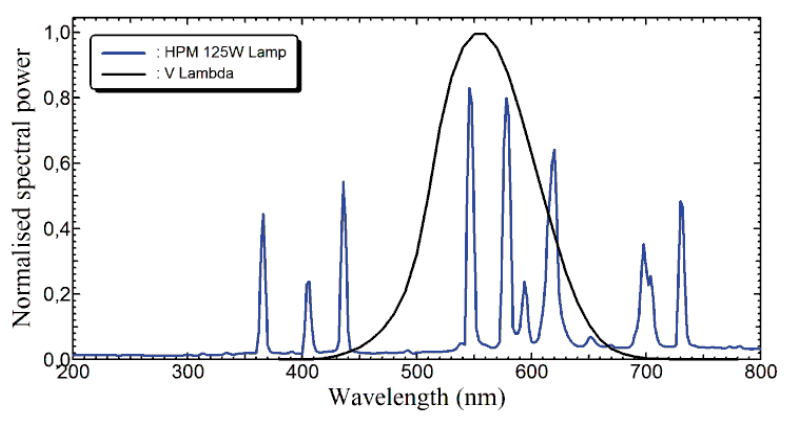

(A)

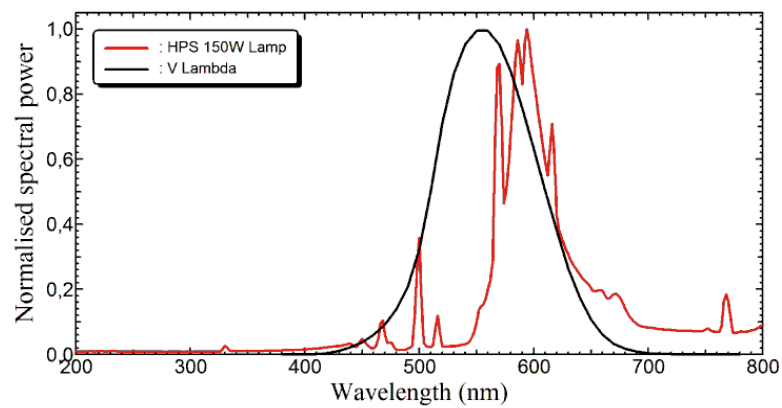

(B)

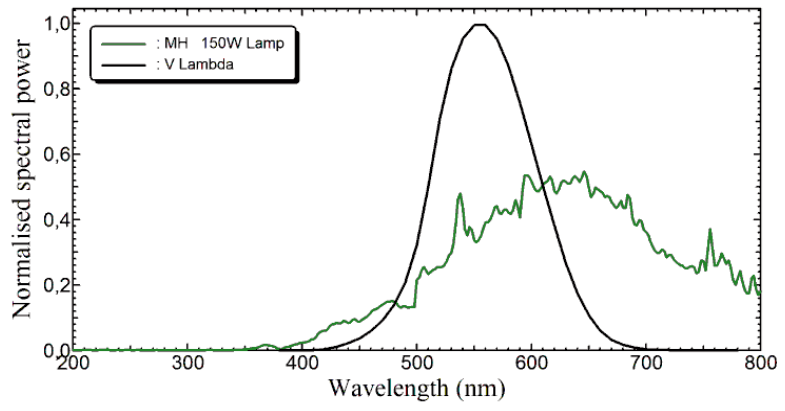

(C)

Fig. 6. Normalized spectral power distribution using monochromator based method of (A) HPM (B) HPS (C) MH lamps.

Evaluation of the uncertainty is done by the Guide to the expression of uncertainty in measurement (GUM) method. This method is adopted and described in details by international organization for standardization (ISO) (ISO, 1993) [12].

The standard uncertainty $u\left(x_{\mathrm{i}}\right)$ to be associated with input quantity $x_{\mathrm{i}}$ is the estimated standard deviation of the mean (ISO, 1993) [13].

$$
u\left(x_{i}\right)=s\left(\bar{X}_{i}\right)=\left(\frac{1}{n(n-1)} \sum_{k=1}^{n}\left(X_{i, k}-\bar{X}_{i}\right)^{2}\right)^{1 / 2} .
$$

The combined standard uncertainty $u_{c}(y)$ is obtained by combining the individual standard uncertainties $u_{i}$, these can be evaluated as Type A and Type B.

That is,

$$
u_{c}^{2}(y)=\sum_{i=1}^{N}\left(\frac{\partial f}{\partial x_{i}}\right)^{2} u^{2}\left(x_{i}\right)
$$


Table 1. Uncertainty accompanied with the normalized spectral power distribution measurements.

\begin{tabular}{ccc}
\hline Uncertainty component & \multicolumn{2}{c}{ Relative standard uncertainty \% } \\
\cline { 2 - 3 } & Type A \% & Type B \% \\
\hline Irradiance responsivity of standard radiometer & & 1.24 \\
Current regulation of lamp & 0.32 & \\
Distance measurements & 0.01 & \\
Repeatability & 0.01 & \\
\hline Expanded uncertainty $(k=2)$ & & $\pm 2.56 \%$ \\
\hline
\end{tabular}

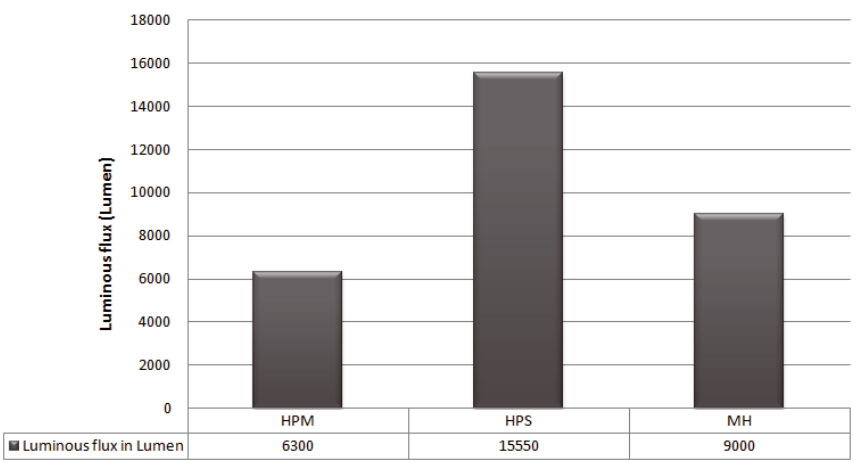

Fig. 7. Lamps absolute luminous flux in lumen using integrating sphere based method.

Uncertainty model used for the determination of the normalized spectral power distribution $E_{S P D}(\lambda)$ is:

$$
E_{S P D}(\lambda)=E_{s}(\lambda)+\delta E_{l}+\delta E_{r}+\delta E_{i},
$$

where,

$E_{s}(\lambda)=$ Uncertainty due to reference spectral irradiance standard radiometer, obtained from the calibration certificate.

$\delta E_{l}=$ Distance effect on the irradiance measurements, Calculated by using the inverse square law.

$\delta E_{r}=$ Repatibility of the measurements, calculated by standard deviation of repeated 5 times.

$\delta E_{i}=$ Uncertianty due to effect of current regulation of lamp on the irradiance measurements, determined by the sensitivity of variation of luminous flux and the current versus $V_{m}$ (main voltage) [10].

On the other hand, uncertainty model used for the determination of the absolute luminous flux measurements is:

$$
F(\lambda)=F_{s}(\lambda)+\delta F_{a}+\delta F_{b}+\delta F_{r}+\delta F_{m}+\delta F_{c},
$$

where,

$F_{s}(\lambda)=$ Uncertainty due to reference luminous flux standard lamp, obtained from the calibration certificate.

$\delta F_{a}=$ Uncertainty due to aging effect determined by the rate of luminous flux drift per hour and the interval between two calibrations (In this work the interval is $50 \mathrm{~h}$ ). $\delta F_{b}=$ Uncertainty due to self absorption is $2 \sigma$ where $\sigma$ is the standard deviation of the values of self absorption correction factor.

$\delta F_{r} \quad=$ Repatibility of the measurements, determined by standard deviation of repeated 5 times.

$\delta F_{m}=$ Uncertainty due to mismatching factor, determined by multiplying the uncertainty of test lamp correlated color temperature $C C T$ with the slope of colour correction factor $c c f$ as function of $C C T$ for such lamp.

$\delta F_{i}=$ Uncertainty due to effect of current regulation of lamp on measurements of flux determined by the sensitivity of variation of luminous flux and the current versus $V_{m}$ (main voltage) [10].

Table 1 gives the relative expanded standard uncertainty accompanied with the normalized spectral power distribution taking by monochromator based method and equal to $\pm 2.56 \%$. Table 2 gives the relative expanded standard uncertainty accompanied with the absolute luminous flux measurements taking by integrating sphere based method and equal to $\pm 2.83 \%$.

\section{Conclusion}

The absolute luminous flux as well as the relative spectral power distribution of three HID outdoor lighting lamps from OSRAM is carried out. Due to different content material inside the arc tube for each type of lamps, the relative spectral power distributions of the three lamps inside $V(\lambda)$ are completely different (see Fig. 6).

The absolute luminous flux measurements from each lamp are obtained, using $V(\lambda)$ standard detector (see Fig. 7). Results show that the HPS has the higher flux according to the integrating sphere based method results with respect to the other lamps. According to monochromator based method, the spectral of sodium lamp has its peaks nearly to $588.9 \mathrm{~nm}$ and $589.5 \mathrm{~nm}$ close to peak of photopic $V(\lambda)(555 \mathrm{~nm})$ and yellow spectra; where the eye sensitivity is high. Hence, they used for traffic road. While the spectral of either HPM or MH lamps, have more wide continuous spectra covering the visible range than HPS lamps, in spite of they have lower flux than HPS; that why they used in the cities for pedestrian. However, $\mathrm{MH}$ lamps have more flux than HPM, so it is preferable to use and has more application in lighting. The measurements were performed under control of environmental conditions 
Table 2. Uncertainty accompanied with the absolute luminous flux measurements.

\begin{tabular}{ccc}
\hline Uncertainty component & \multicolumn{2}{c}{ Relative standard uncertainty \% } \\
\cline { 2 - 3 } & Type A \% & Type B \% \\
\hline Calibration of luminous flux standard lamp & & 0.7 \\
Aging of the working standard lamp & 0.5 \\
$\quad$ Self absorption correction & 0.9 & \multirow{2}{*}{0.53} \\
Spectral mismatch correction & 0.39 \\
Repeatability of test lamp & 0.17 \\
Current regulation of lamp & \multicolumn{3}{c}{ $\pm 2.83 \%$} \\
\hline Expand uncertainty at $(k=2)$ & \multicolumn{2}{c}{} \\
\hline
\end{tabular}

and good regulation of electrical power. The accompanied standard uncertainties with the measurements are evaluated. It is equal to $\pm 2.56 \%$ with the normalized spectral power distribution measurements and $\pm 2.83 \%$ with the absolute luminous flux measurements.

\section{References}

1. C. Decusatis, Handbook of applied photometry (American institute of physics (AIP), New York, 1997)

2. D. Schreuder, Outdoor lighting: Physics, vision and perception (Springer, Heidelberg, 2008)

3. Ringorrango importation of products [homepage on the internet], Spain, http://www.ringorrango.es/

4. E.J. Ientilucci, Measurement of the spectral distribution of gas discharge, fluorescent and incandescent sources (Rochester Institute of Technology, College of Science, Center for Imaging Science, Digital Imaging and Remote Sensing Laboratory, New York, 2000)

5. Commercial lighting company [homepage on the internet], Florida, http://www.clcbulbs.com/
6. A.A. Abd-Elmageed, Detector-based traceability chain for spectral irradiance using tunable laser based facility at PTB [dissertation] (Braunschweig University, Germany, 2011)

7. IES guide to lamp seasoning (Illuminating Engineering Society (IES), New York, 2012)

8. S.M. Reda, A.A. Abd-Elmageed, On the use of spectral Lamp lines in the Monochromator Calibration. J. Trends Adv. Sci. Eng. 4, 22-25 (2012)

9. Illuminance meter LMT-B520 manual, Lichtmesstechnik (LMT) company, Berlin

10. E.M. El-moghazy, A technical study of the development of working standard lamps for lighting applications [dissertation] (Ein Shames University, Egypt, 2012)

11. CIE Standard Colorimetric Observers (International Commission on Illumination (CIE), Vienna, 2006)

12. Guide to the expression of uncertainty in measurement $(G U M)$ (International Organization for Standardization (ISO), Switzerland, 1995)

13. The expression of uncertainty and confidence in measurement, 3rd edn. (United Kingdom Accreditation Service (UKAS), United Kingdom, 2012) 